Article

\title{
Identification of Two Porcine Reproductive and Respiratory Syndrome Virus Variants Sharing High Genomic Homology but with Distinct Virulence
}

\author{
Nanhua Chen ${ }^{1, *}$, Mengxue Ye ${ }^{1}$, Yucheng Huang ${ }^{1}$, Shuai $\mathrm{Li}^{1}$, Yanzhao Xiao ${ }^{1}$, Xinshuai Li ${ }^{1}$, \\ Shubin $\mathrm{Li}^{1}{ }^{1}$, Xiangdong $\mathrm{Li}^{2}$, Xiuling $\mathrm{Yu}^{2}$, Kegong Tian ${ }^{2}$ and Jianzhong $\mathrm{Zhu}{ }^{1, *}$ \\ 1 College of Veterinary Medicine, Yangzhou University, Yangzhou, Jiangsu 225009, China \\ 2 National Research Center for Veterinary Medicine, High-Tech District, Luoyang, Henan 471003, China \\ * Correspondence: chnhlh@126.com (N.C.); jzzhu@yzu.edu.cn (J.Z.)
}

Received: 1 August 2019; Accepted: 17 September 2019; Published: 18 September 2019

\begin{abstract}
Porcine reproductive and respiratory syndrome virus (PRRSV) causes huge economic loss to the global swine industry. Even though several control strategies have been applied, PRRS is still not effectively controlled due to the continuous emergence of new variants and limited cross-protection by current vaccines. During the routine epidemiological investigation in 2017, two PRRSV variants were identified from a severe abortion farm and a clinically healthy farm, respectively. The viruses were isolated and denominated as XJ17-5 and JSTZ1712-12. Genomic sequencing indicated that their genomes are both 14,960 bp in length sharing $99.45 \%$ nucleotide identity. Sequence alignments identified a discontinuous 30-amino-acid deletion and a continuous 120-amino-acid deletion in nsp2 of both isolates. Genome-based phylogenetic analysis confirmed that XJ17-5 and JSTZ1712-12 belong to the HP-PRRSV subtype but form a new branch with other isolates containing the same 150 -amino-acid deletion in $n s p 2$. Pathogenic analysis showed that XJ17-5 is highly virulent causing $60 \%$ mortality, while JSTZ1712-12 is avirulent for piglets. Furthermore, fragment comparisons identified 34-amino-acid differences between XJ17-5 and JSTZ1712-12 that might be associated with the distinct virulence. The identification of highly homologous HP-PRRSV variants with new genetic feature and distinct virulence contributes to further analyze the pathogenesis and evolution of PRRSV in the field.
\end{abstract}

Keywords: Porcine reproductive and respiratory syndrome virus; high homology; deletion; distinct virulence; potential virulence-associated amino acids

\section{Introduction}

Porcine reproductive and respiratory syndrome (PRRS) has been a critical economic disease in the global swine industry for three decades. The annual cost caused by PRRSV was estimated to be $\$ 664$ million in national breeding and growing pig herds in the United States alone [1]. PRRSV causes severe reproductive failure in pregnant sows and respiratory distress in neonatal pigs [2]. The etiological agent, PRRS virus (PRRSV), is an enveloped, positive-sense, single-stranded RNA virus classifying within the family Arteriviridae [3]. PRRSV genome is $\sim 15 \mathrm{~kb}$ in length and encodes at least 10 open reading frames (ORFs), including ORF1a, ORF1b, ORF2a, ORF2b, ORF5 $a$ and ORF3-7. ORF1a and $O R F 1 b$ encode at least 16 nonstructural proteins (nsps) that are critical for viral replication and transcription, while ORF2-7 encode eight structural proteins to constitute the viral particle [3,4].

PRRSV is one of the most rapidly evolving RNA viruses [5]. Different PRRSV isolates are antigenically, genetically and pathologically distinct [6]. PRRSV isolates can be divided into PRRSV1 and PRRSV2 species [7]. In Chinese swine herds, PRRSV2 isolates are predominant, while PRRSV1 
isolates may also be sporadically detected [8,9]. Three major subtypes of PRRSV2 isolates co-exist in Chinese swine herds including classical PRRSV2, highly pathogenic PRRSV (HP-PRRSV) and NADC30-like PRRSV [10-12]. Classical PRRSV2 isolates (CH-1a, S1 and BJ-4) that first emerged in China in 1996 are low virulent $[10,13]$. HP-PRRSV isolates (JXA1, HuN4 and TJ) first emerged in China in 2006, which are characterized by high fever $\left(40-42^{\circ} \mathrm{C}\right)$, high morbidity $(50-100 \%)$ and high mortality $(20-100 \%)$ in all ages of pigs $[11,14,15]$. HP-PRRSV isolates have a genetic hallmark of discontinuous 30 -amino-acid deletion in $n s p 2$, but it is not related to the high virulence of HP-PRRSV [16]. NADC30-like PRRSV isolates (JL580, CHsx1401 and HNjz15) first emerged in China in 2013 [12]. Their virulence is quite variable but relatively lower than HP-PRRSV isolates [17]. The unique genetic feature of NADC30-like PRRSV isolates is a discontinuous 131-amino-acid deletion in nsp2 [12,18,19].

In this study, two novel HP-PRRSV variants were isolated from a diseased pig from Xinjiang province and a healthy pig from Jiangsu province in 2017. Both HP-PRRSV variants have the discontinuous 30-amino-acid deletion and an additional 120-amino-acid deletion in nsp2. Genomic sequencing identified that they share high genomic homology. Intriguingly, animal challenge study showed that they have distinct virulence.

\section{Materials and Methods}

\subsection{Sample Collection and Differential Detection}

In April 2017, an outbreak characterized by high fever and severe abortions occurred in a large-scale pig farm with $\sim 4500$ sows in Xinjiang province, China. The outbreak caused $>1000$ abortions and the deaths of $>100$ sows and $>10,000$ weaned piglets within half a year. To investigate the causative agent for the outbreak, a total of 17 sera from fever piglets and 9 lungs from diseased and euthanized piglets were frozen and submitted to the Animal Hospital at Yangzhou University. In December 2017, a total of 18 sera were submitted from a clinically healthy pig farm with 200 sows in Taizhou city, Jiangsu province, China for routine epidemiological investigation. The sera and lungs were used as templates for the routine detection of common swine viruses including PRRSV, classical swine fever virus (CSFV), porcine epidemic diarrhea virus (PEDV), pseudorabies virus (PRV), porcine parvovirus (PPV) and porcine circovirus 2 (PCV2) by conventional and real-time RT-PCR assays [9,20-22].

\subsection{Virus Isolation and Genome Sequencing}

To further analyze the novel PRRSV variants identified in this study, a positive lung homogenate from Xinjiang province and a positive serum sample from Jiangsu province were used for virus isolation in Marc-145 cells as previously described [23]. Briefly, the tissue homogenate and the serum sample were inoculated in Marc- 145 cells, respectively. The inoculated cells were maintained at $37^{\circ} \mathrm{C}$ in a $5 \% \mathrm{CO}_{2}$ atmosphere and monitored daily for cytopathic effects (CPE). The cultures were frozen at $-80{ }^{\circ} \mathrm{C}$ when approximately $70 \% \mathrm{CPE}$ was reached or after 7 days of culturing. The resultant viruses were denominated as XJ17-5 and JSTZ1712-12, respectively. The isolation of PRRSV was confirmed by the indirect immunofluorescence assay (IFA) staining. Marc- 145 cells were infected with 200 median tissue culture infectious doses (TCID 50 ) of JSTZ1712-12 and XJ17-5, respectively. The infected Marc- 145 cells were fixed at $24 \mathrm{~h}$ post infection and evaluated by IFA according to the procedure described previously [24]. PRRSV-specific murine mAb 15A1 (1:500 dilution) against the N protein was used as the primary antibody, while the Dylight 594 (Goat anti-mouse IgG, 1:1000, Invitrogen, Carlsbad, CA, USA) was used as the secondary antibody.

Total RNA was extracted from the cell culture using an RNeasy Mini Kit (Qiagen, Hilden, Germany) according to the manufacturer's instructions. The complete genomes were determined using ten pairs of primers amplifying overlapped fragments as previously reported [23] (Table S1). The amplicons were purified with an E.Z.N.A. Gel Extraction Kit (Omega, Mansfield, TX, USA) and cloned into pEASY-T1 Vector (Transgen, Beijing, China). At least three recombinant clones for each fragment were sequenced by the GENEWIZ Company (Suzhou, China). The obtained sequences were assembled by 
the DNAMAN 6.0 software and the complete genomes of XJ17-5 and JSTZ1712-12 were deposited in the GenBank database with the accession numbers of MK759853 and MK906026, respectively.

\subsection{Multiple Alignments, Phylogenetic and Recombination Analyses}

To determine the similarity between XJ17-5 and JSTZ1712-12 isolates, the complete genome and each fragment alignments were performed using the DNAMAN 6.0. To analyze the evolutionary relationship between our isolates and other Chinese isolates, a total of 50 representative PRRSV genomes were obtained from the GenBank database and multiplex sequence alignment was generated using Clustal X [25]. Genome-based phylogenetic analysis was performed using MEGA 6.06 [26]. Phylogenetic tree was constructed from aligned genomes using the neighbor-joining method as previously described [27]. The robustness of the phylogenetic tree was evaluated by bootstrapping using 1000 replicates. In addition, the potential recombination events in XJ17-5 and JSTZ1712-12 isolates were screened using the aligned genomes by RDP4 and SimPlot 3.5.1 as previously described [23,28,29]. Briefly, seven methods embedded in RDP4 software, including RDP, GENECONV, BootScran, Maxchi, Chimaera, SiScan and 3Seq were used to detected recombination events and their beginning and ending breakpoints. The default settings were used for all the seven methods, and the highest acceptable $p$ value was set at 0.05 . In addition, the detected recombination events were further confirmed by SimPlot 3.5.1 using a potential recombinant virus as the query virus and the potential parental viruses as the reference viruses.

\subsection{Animal Challenge Study}

Considering that XJ17-5 and JSTZ1712-12 isolates shared high genomic homology but were isolated from clinically diseased and healthy pigs, respectively, we determined their virulence by animal challenge study. The challenge study was approved (April 7, 2018) by the Animal Welfare and Ethics Committee at College of Veterinary Medicine of Yangzhou University with the reference number of YZU-CVM-201806. Fifteen 4-week-old PRRSV-free piglets were randomly divided into three groups (five piglets per group). Piglets in two groups were intranasally and intramuscularly inoculated with $2 \mathrm{~mL} 10^{5.0}$ median tissue culture infectious doses $\left(\mathrm{TCID}_{50}\right) / \mathrm{mL}$ XJ17-5 (passage 3 ) and JSTZ1712-12 (passage 3), respectively, while piglets in the third group were inoculated with Minimum Essential Medium Eagle (MEM media) to serve as the negative control.

Rectal temperature and clinical signs were recorded daily. Serum samples were collected at 0, 4, 7, 11 and 14 days post infection (dpi) for the analyses of virus load and antibody level. The dynamics of viremia were analyzed by real-time RT-PCR [22]. PRRSV-specific antibodies in the sera were detected by HerdCheck ${ }^{\circledR}$ PRRS $\times 3$ ELISA Kit (IDEXX, Westbrook, ME, USA). The threshold for seroconversion was set at sample-to-positive ( $\mathrm{s} / \mathrm{p})$ ratio of 0.4 according to the manufacture's instruction. The pigs survived until $14 \mathrm{dpi}$ were euthanized and tissue samples were collected for histopathological and immunohistochemical examinations [11,23].

\subsection{Statistical Analysis}

The data of rectal temperature, virus load, antibody level and body weight were shown in means \pm standard deviations (SD). The differences between groups were determined by Mann-Whitney $U$ Test using Graphpad Prism version 6.07 [23]. A $p$ value $<0.05$ was considered statistically significant.

\section{Results}

\subsection{Clinical Sample Detection}

Twelve out of 17 sera and 6 out of 9 lungs from Xinjiang province were detected as PRRSV positive, and 2 out of 18 sera from Jiangsu province were detected as PRRSV positive, while all the other pathogens were not detected. ORF5 sequencing showed that PRRSV from all Xinjiang positive samples shared $100 \%$ nucleotide identity and the two Jiangsu positive samples also shared $100 \%$ 
nucleotide identity. Remarkably, ORF5 sequences from the positive samples from Xinjiang and Jiangsu provinces shared $99.83 \%$ nucleotide identity. In addition, $n s p 2$ sequencing showed that their $n s p 2$ shared 99.33\% nucleotide identity. The high similarities in both PRRSV most variable genes (ORF5 and nsp2) suggested that the viruses from clinically diseased and healthy pig farms are highly homologous.

\subsection{Virus Isolation and Growth Curve}

The XJ17-5 and JSTZ1712-12 viruses were successfully isolated in Marc-145 cells. Typical PRRS-specific CPE could be observed at $\sim 4$ dpi. The presence of PRRSV was confirmed by the IFA staining. PRRSV-specific fluorescence could be observed at $24 \mathrm{~h}$ post infection in both XJ17-5 and JSTZ1712-12 infected Marc-145 cells but not in mock infected Marc-145 cells (Figure 1). In addition, one-step growth curves of XJ17-5 and JSTZ1712-12 in pulmonary alveolar macrophages (PAM) and Marc-145 cells were also determined, which showed that the replication efficacies of XJ17-5 and JSTZ1712-12 in vitro are not significantly different $(p>0.05)$ (Figure 2). The area under the curves (AUC) for virus load versus time were also calculated as previously described [30,31]. The AUC values between XJ17-5 and JSTZ1712-12 infections in either PAM or Marc-145 cells are similar $(p>0.05)$ (Table S2), which further supported that XJ17-5 and JSTZ1712-12 strains have similar replication efficacies.
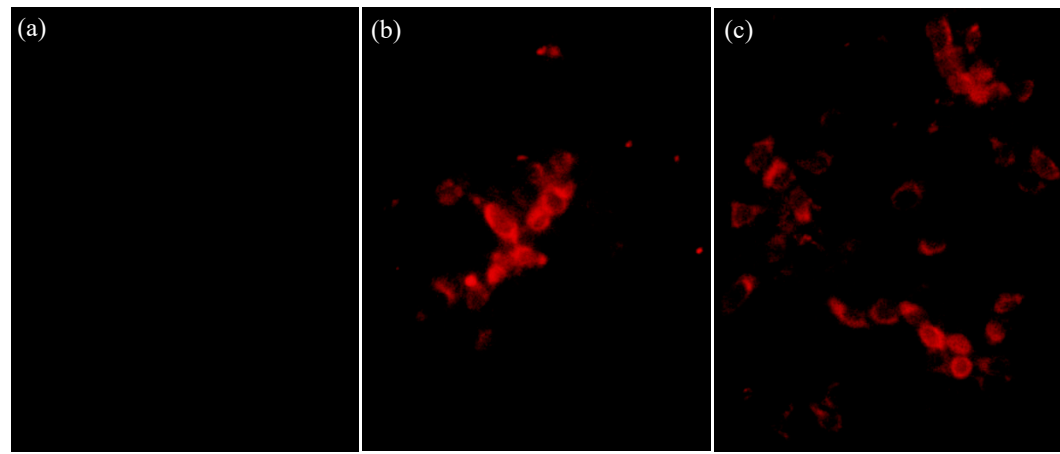

Figure 1. Identification of XJ17-5 and JSTZ1712-12 isolates in Marc-145 cells by the immunofluorescence assay (IFA) staining. Marc-145 cells were infected with 200 median tissue culture infectious doses (TCID 50 ) of XJ17-5 and JSTZ1712-12, respectively. The infected Marc-145 cells were fixed at $24 \mathrm{~h}$ post infection and evaluated by IFA according to the standard procedure [24]. Porcine reproductive and respiratory syndrome virus (PRRSV)-specific murine mAb 15A1 (1:500 dilution) against the N protein was used as the primary antibody, while the Dylight 594 (Goat anti-mouse IgG, 1:1000, Invitrogen) was used as the secondary antibody. PRRSV-specific antigen could not be detected in mock infected (a) Marc-145 cells but could be detected in XJ17-5-infected (b) and JSTZ1712-12-infected (c) Marc-145 cells. Original magnification at $200 \times$.
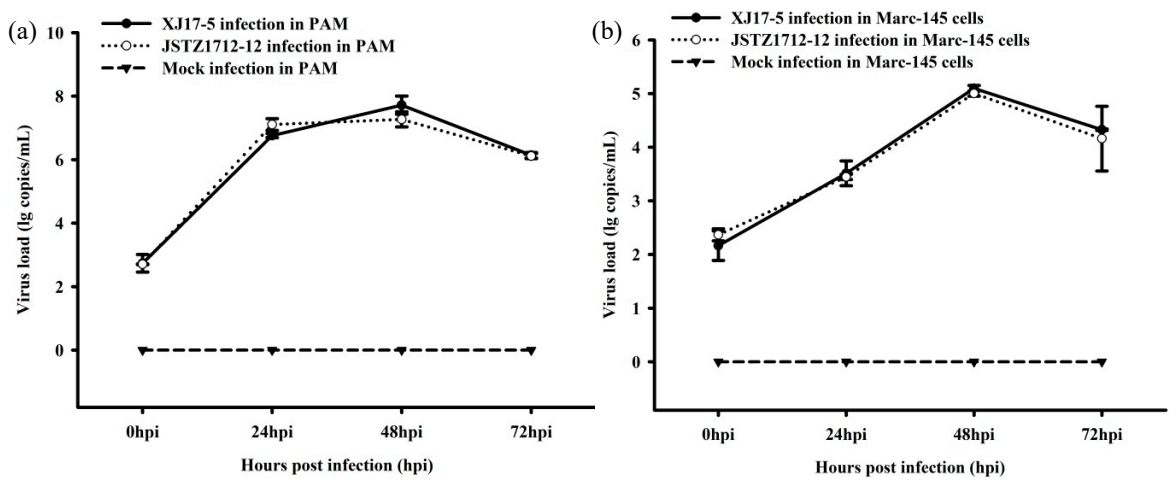

Figure 2. The in vitro replication of JSTZ1712-12 and XJ17-5 isolates. The one-step growth curves in pulmonary alveolar macrophages (PAM) (a) and Marc-145 cells (b) within $72 \mathrm{~h}$ post infection (hpi) were determined by real-time RT-PCR assay [22]. No significant difference was detected in in vitro replication of JSTZ1712-12 and XJ17-5 isolates. 


\subsection{Genomic Comparison}

The complete genomes of XJ17-5 and JSTZ1712-12 isolates were determined, which are both 14,960 bp excluding poly (A) tail. XJ17-5 and JSTZ1712-12 genomes share $99.45 \%$ nucleotide identity. Genomic comparison with other representative PRRSV strains showed that XJ17-5 and JSTZ1712-12 share $58.31 \% / 58.31 \%, 86.27 \% / 86.45 \%, 82.13 \% / 81.93 \%$ and $96.46 \% / 96.84 \%$ nucleotide identities with representative strains of PRRSV1 (Lelystad virus, LV, M96262), classical PRRSV2 (ATCC VR-2332, PRU87392), NADC30-like PRRSV (NADC30, JN654459) and HP-PRRSV (TJ, EU860248), respectively (Table 1). Each fragment comparison also showed that XJ17-5 and JSTZ1712-12 isolates share the highest nucleotide identity with the HP-PRRSV strain. Both XJ17-5 and JSTZ1712-12 isolates were not detected as recombinant viruses by either RDP4 or Simplot 3.5.1 [23,28,29]. In addition, each fragment alignment identified that both XJ17-5 and JSTZ1712-12 isolates have the discontinuous 30-amino-acid deletion at 481 and 533-561 positions of $n s p 2$, which is the genetic hallmark of HP-PRRSV. Remarkably, they also have a continuous 120 amino-acid deletion at 628-747 positions of $n s p 2$ (Figure 3). The results indicated that XJ17-5 and JSTZ1712-12 isolates are novel HP-PRRSV variants.

Table 1. Detailed comparison of XJ17-5/JSTZ1712-12 genomes to representative PRRSV strains.

\begin{tabular}{|c|c|c|c|c|c|}
\hline \multirow{2}{*}{ XJ17-5/JSTZ1712-12 * } & \multirow{2}{*}{ XJ17-5/JSTZ1712-12 \# } & VR-2332 & NADC30 & TJ & LV \\
\hline & & \multicolumn{4}{|c|}{ Nucleotide Identity (\%) to XJ17-5/JSTZ1712-12 } \\
\hline Complete (14960) & 99.45 & $86.27 / 86.45$ & $82.13 / 81.93$ & $96.46 / 96.84$ & $58.31 / 58.31$ \\
\hline 5'UTR (1-189) & 99.47 & $91.01 / 91.53$ & $90.58 / 91.10$ & $96.30 / 96.83$ & $51.80 / 53.15$ \\
\hline ORF1a (190-7251) & 99.35 & $82.00 / 82.31$ & $72.55 / 72.65$ & $93.82 / 94.25$ & $53.30 / 53.31$ \\
\hline Nsp1 $\alpha(190-729)$ & 99.63 & $92.04 / 92.04$ & $89.81 / 90.00$ & $99.26 / 99.63$ & $62.11 / 61.92$ \\
\hline Nsp1 $\beta(730-1338)$ & 98.36 & $86.21 / 86.54$ & $80.62 / 80.79$ & $98.03 / 99.01$ & $47.76 / 47.92$ \\
\hline Nsp2 (1339-4479) & 99.33 & $73.88 / 74.21$ & $67.26 / 67.30$ & $88.17 / 88.60$ & $49.44 / 49.39$ \\
\hline Nsp2N (1339-3438) & 99.14 & $66.31 / 66.75$ & $58.31 / 58.54$ & $83.41 / 83.98$ & $41.27 / 41.16$ \\
\hline $\begin{array}{c}\text { Nsp2TF (1339-3438, } \\
3441-3945)\end{array}$ & 99.31 & $70.92 / 71.82$ & $63.97 / 64.22$ & $86.11 / 86.59$ & $47.37 / 47.17$ \\
\hline Nsp3 (4477-5166) & 98.99 & $88.55 / 89.28$ & $82.03 / 82.46$ & $97.83 / 98.55$ & $56.23 / 56.52$ \\
\hline Nsp4 (5167-5778) & 99.67 & $89.87 / 89.87$ & $85.29 / 84.97$ & $99.67 / 99.67$ & $60.62 / 60.95$ \\
\hline Nsp5 (5779-6288) & 99.61 & $89.02 / 89.41$ & $90.20 / 90.59$ & $99.02 / 99.41$ & $63.14 / 63.33$ \\
\hline Nsp6 (6289-6336) & 100 & $93.75 / 93.75$ & $91.67 / 91.67$ & $97.92 / 97.92$ & $70.83 / 70.83$ \\
\hline Nsp7 $\alpha(6337-6783)$ & 99.55 & $90.38 / 90.83$ & $84.56 / 84.56$ & $98.88 / 99.33$ & $55.36 / 55.58$ \\
\hline$N s p 7 \beta(6784-7113)$ & 100 & $87.88 / 87.88$ & $79.39 / 79.39$ & $99.39 / 99.39$ & $48.66 / 48.66$ \\
\hline Nsp8 (7114-7248) & 100 & $96.30 / 96.30$ & $89.63 / 89.63$ & $100 / 100$ & $64.44 / 64.44$ \\
\hline ORF1b (7248-11621) & 99.47 & $91.08 / 91.06$ & $87.93 / 87.86$ & $98.88 / 99.22$ & $62.11 / 62.14$ \\
\hline Nsp9 (7248-9167) & 99.32 & $92.45 / 92.29$ & $87.60 / 87.34$ & $98.54 / 98.91$ & $66.35 / 66.35$ \\
\hline Nsp10 (9168-10487) & 99.39 & $89.85 / 90.00$ & $85.76 / 85.91$ & $98.79 / 99.24$ & $60.30 / 60.30$ \\
\hline Nsp11 (10488-11159) & 99.70 & $90.18 / 90.18$ & $91.22 / 91.22$ & $99.26 / 99.55$ & $65.33 / 65.63$ \\
\hline Nsp12 (11160-11618) & 100 & $89.39 / 89.39$ & $89.83 / 89.83$ & $99.13 / 99.13$ & $46.15 / 46.27$ \\
\hline ORF2a (11623-12393) & 99.74 & $93.13 / 93.39$ & $86.64 / 86.38$ & $99.35 / 99.61$ & $62.13 / 63.55$ \\
\hline ORF2b (11628-11849) & 100 & $93.24 / 93.24$ & $87.67 / 87.67$ & $100 / 100$ & $68.72 / 68.72$ \\
\hline ORF3 (12246-13010) & 99.74 & $89.15 / 89.41$ & $83.14 / 83.40$ & $98.95 / 99.22$ & $61.92 / 62.17$ \\
\hline ORF4 (12791-13327) & 99.63 & $89.76 / 90.13$ & $86.78 / 87.15$ & $99.07 / 99.44$ & $64.49 / 64.67$ \\
\hline ORF5 (13338-13940) & 99.83 & $89.05 / 88.89$ & $86.24 / 86.07$ & $99.17 / 99.34$ & $61.33 / 61.17$ \\
\hline ORF5a (13328-13468) & 100 & $88.65 / 88.65$ & $85.82 / 85.82$ & $98.58 / 98.58$ & $55.32 / 55.32$ \\
\hline ORF6 (13925-14449) & 99.62 & $95.43 / 95.05$ & $89.33 / 88.95$ & $99.43 / 99.81$ & $69.14 / 69.14$ \\
\hline ORF7 (14439-14810) & 98.92 & $93.28 / 93.55$ & $90.32 / 90.59$ & $98.92 / 99.46$ & $62.37 / 60.86$ \\
\hline 3'UTR (14811-14960) & 98.67 & $92.05 / 93.38$ & $88.08 / 89.40$ & $98.67 / 100$ & $55.63 / 54.30$ \\
\hline
\end{tabular}

* The lengths of the complete genome and each fragment of XJ17-5 isolate (MK759853) and JSTZ1712-12 isolate (MK906026) are identical. \# XJ17-5 and JSTZ1712-12 isolates share the highest nucleotide identity with each other at the complete genome and each fragment rather than any other representative PRRSV strains including VR-2332 (PRU87392), NADC30 (JN654459), TJ (EU860248) and Lelystad virus (LV) (M96262). 


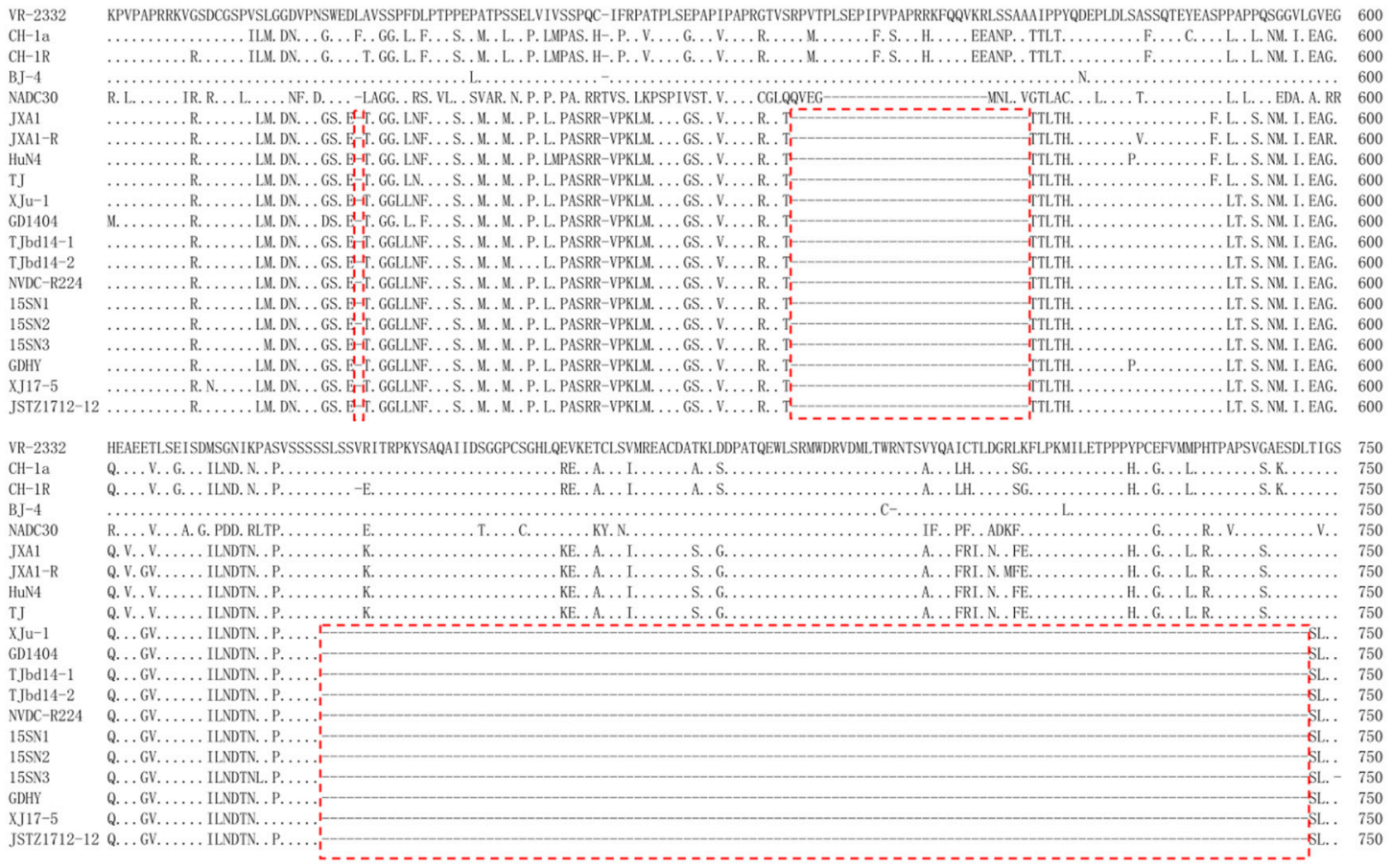

Figure 3. Alignment of $n s p 2$ amino acid sequences to show the 30-amino-acid discontinuous deletion and the 120-amino-acid continuous deletion. The new genetic feature of 150-amino-acid deletion in XJ17-5 and JSTZ1712-12 isolates and other 9 HP-PRRSV variants are shown in red dashed boxes.

\subsection{Phylogenetic Analysis}

Fifty-genome-based phylogenetic analysis further supported that XJ17-5 and JSTZ1712-12 are clustered within the HP-PRRSV subtype. However, they formed a new branch with another nine Chinese HP-PRRSV variants (Figure 4). Remarkably, these 11 HP-PRRSV variants all have the same 30-amino-acid discontinuous deletion and 120-amino-acid continuous deletion within nsp2 (Figure 3). These variants were isolated from five provinces/cities (Xinjiang, Guangdong, Shaanxi, Jiangsu, Tianjin) of China in 2012-2017, suggesting that this new group of HP-PRRSV variants has been circulating in Chinese swine herds for several years.

\subsection{Pathogenic Analysis}

Clinical signs including dyspnea, anorexia and diarrhea were observed in XJ17-5-infected pigs but not in JSTZ1712-12-infected or mock-infected pigs. The rectal temperature of JSTZ1712-12-infected and mock-infected pigs was always lower than $40^{\circ} \mathrm{C}$ during the period of animal challenge study; however, the rectal temperature of all five XJ17-5-infected pigs reached $\geq 40{ }^{\circ} \mathrm{C}$ (the highest, $41.2{ }^{\circ} \mathrm{C}$ ) during the challenge study (Figure 5a). Three XJ17-5-infected pigs died at $11 \mathrm{dpi}, 13 \mathrm{dpi}$ and $14 \mathrm{dpi}$, respectively, while all JSTZ1712-12-infected and mock-infected pigs survived healthy until the end of the study (Figure 5b). Viremia and PRRSV-specific antibody could be detected in all XJ17-5-infected and JSTZ1712-12-infected pigs since $7 \mathrm{dpi}$ and $11 \mathrm{dpi}$, respectively (Figure $5 \mathrm{c}, \mathrm{d}$ ). The XJ17-5-infected pigs had significantly lower body weight than mock-infected pigs at $11 \mathrm{dpi}$, but there was no significant difference in body weights between JSTZ1712-12-infected pigs and mock-infected pigs (Figure 5e). 


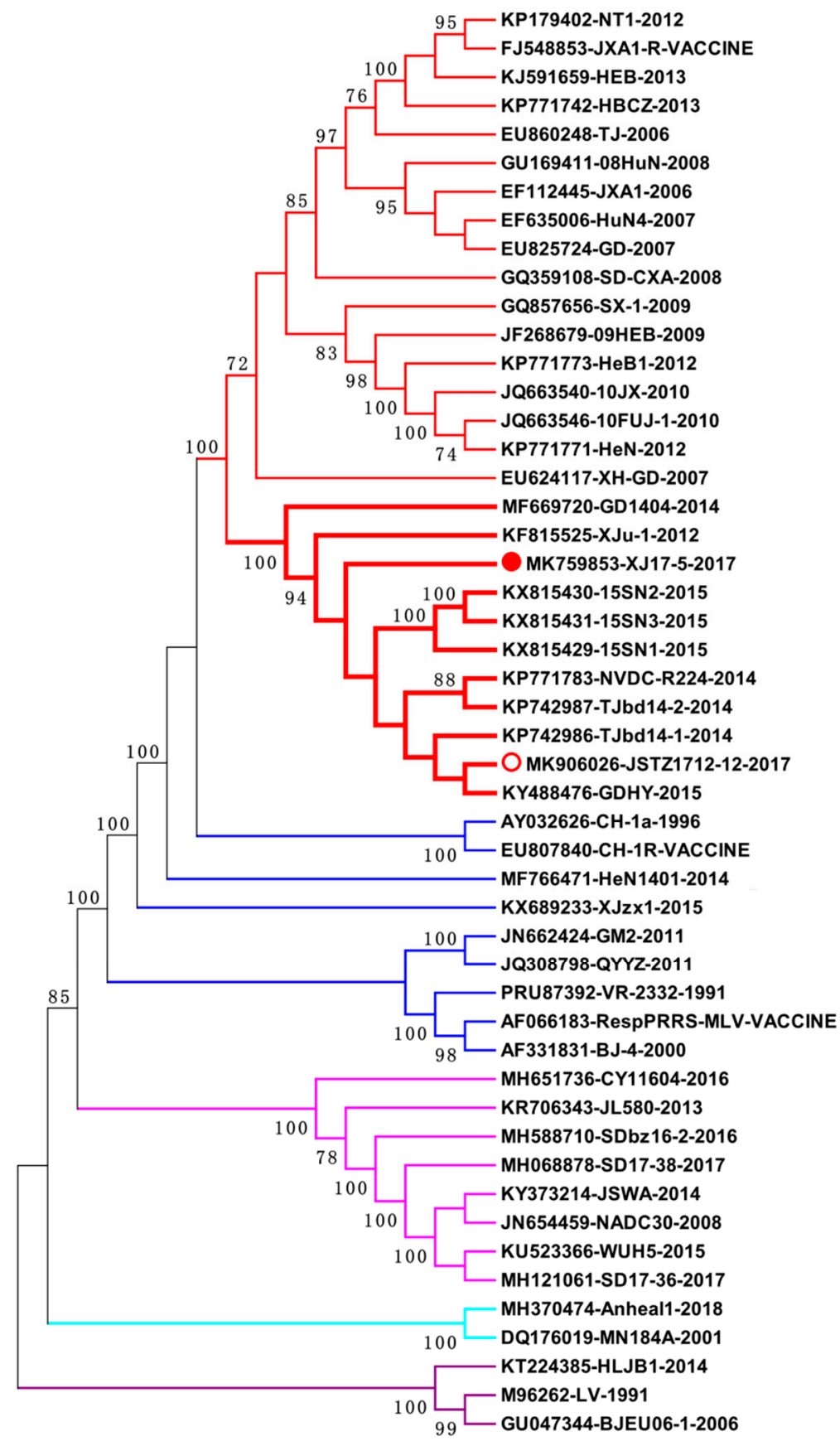

Figure 4. Genome-based genotyping based on 50 representative PRRSV genomes using MEGA 6.06. Our XJ17-5 and JSTZ1712-12 isolates are clustered within the HP-PRRSV subtype and form a new branch with another nine HP-PRRSV variants containing the same 150-amino-acid deletion in $n s p 2$. Different types/subtypes are shown in different colors. XJ17-5 and JSTZ1712-12 are highlighted with solid and empty red circles, respectively. The new branch formed by XJ17-5, JSTZ1712-12 and another nine HP-PRRSV variants containing the same 150 -amino-acid deletion in $n s p 2$ is shown in bold. Each virus is presented by the Genbank accession number, the virus name and the year of isolation. Bootstrap values from 1000 replications are indicated for each node, while bootstrap values $<70$ were not shown. 

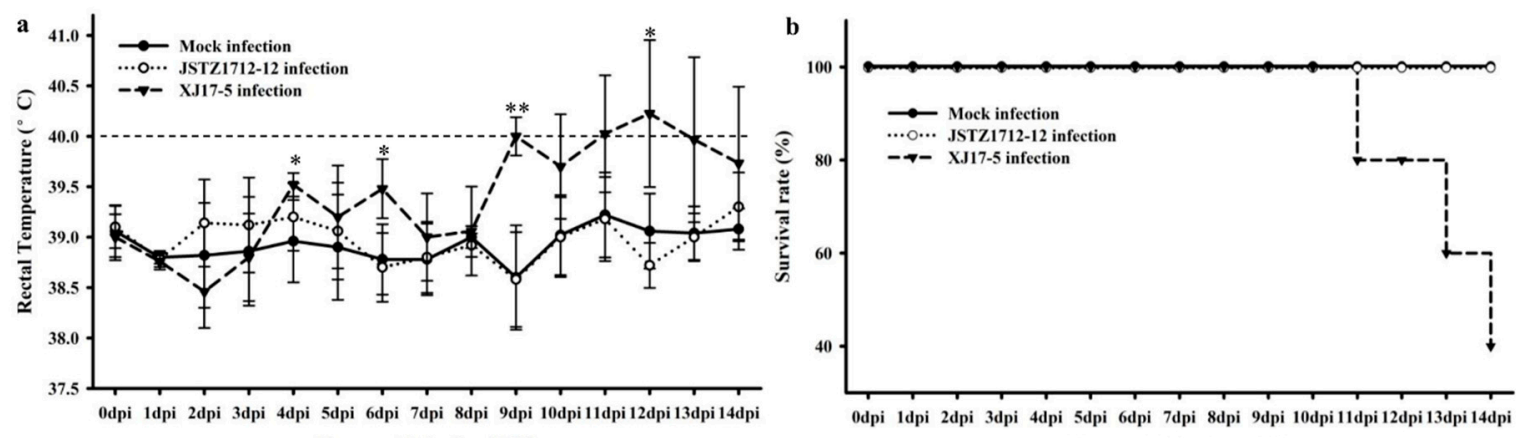

Days post infection (DPI)
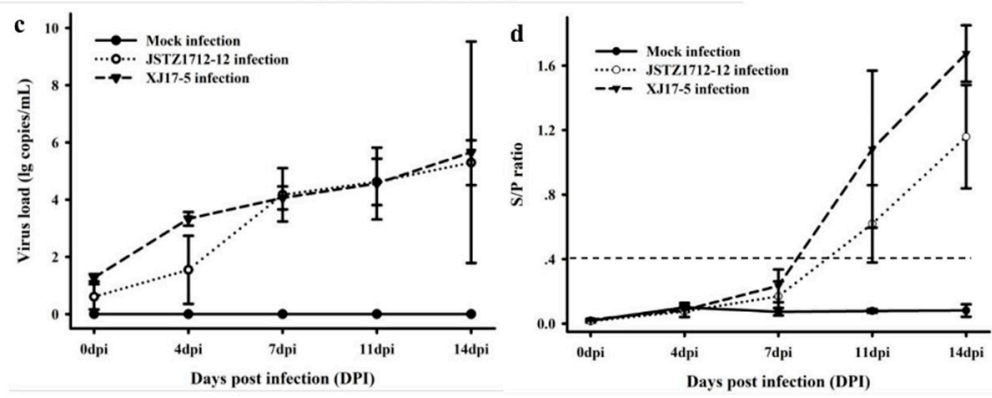
Days post infection (DPI)

Figure 5. Dynamics of rectal temperature, survival rate, virus load, antibody level and body weight during the challenge study. XJ17-5-infected pigs showed high fever (all pigs $\geq 40{ }^{\circ} \mathrm{C}$ with the highest being $41.2^{\circ} \mathrm{C}$ ), while JSTZ1712-12-infected and mock-infected pigs were all lower than $40{ }^{\circ} \mathrm{C}$. The significantly higher rectal temperature in XJ17-5-infected pigs was shown with the asterisk $\left(^{*}: p<0.05\right.$, **: $p<0.01$ ) (a). XJ17-5 infection resulted in 60\% mortality within $14 \mathrm{dpi}$, while JSTZ1712-12-infected and mock-infected pigs all survived during the challenge study $(\mathbf{b})$. Viremia was analyzed using the real-time RT-PCR assay [22]. The virus could be detected in all XJ17-5-infected pigs and JSTZ1712-12-infected pigs from $4 \mathrm{dpi}$ and $7 \mathrm{dpi}$, but not in mock-infected pigs (c). PRRSV-specific antibody was detected by IDEXX HerdCheck ${ }^{\circledR}$ PRRS $\times 3$ Antibody Detection ELISA kit. The threshold for seroconversion was set at sample-to-positive (s/p) ratio of 0.4. PRRSV-specific antibody could be detected in all XJ17-5-infected and JSTZ1712-12-infected pigs from $11 \mathrm{dpi}(\mathbf{d})$. XJ17-5-infected pigs have significantly lower body weight than the mock-infected pigs at $11 \mathrm{dpi}(p<0.05)$, while JSTZ1712-12-infected pigs have no significantly difference in body weight comparing with the mock-infected pigs (e).

During necropsy examination, lung consolidation and extensive hemorrhages were observed in XJ17-5-infected pigs but not in JSTZ1712-12-infected or mock-infected pigs (Figure 6a-c). In the histopathological examination, red blood cells and serous exudation were obviously observed only in XJ17-5-infected pigs (Figure 6f). In addition, interstitial pneumonia with infiltration of lymphocytes and macrophages could also be observed in the lungs of XJ17-5-infected (severe) and JSTZ1712-12-infected (mild) pigs but not in mock-infected pigs (Figure 6d-f). PRRSV antigens could be detected in both JSTZ1712-12-infected and XJ17-5-infected pigs but not in mock-infected pigs in the immunohistochemical examination (Figure $6 \mathrm{~g}-\mathrm{i}$ ). The challenge study indicated that XJ17-5 isolate is highly pathogenic but JSTZ1712-12 isolate is nearly not pathogenic for piglets. 

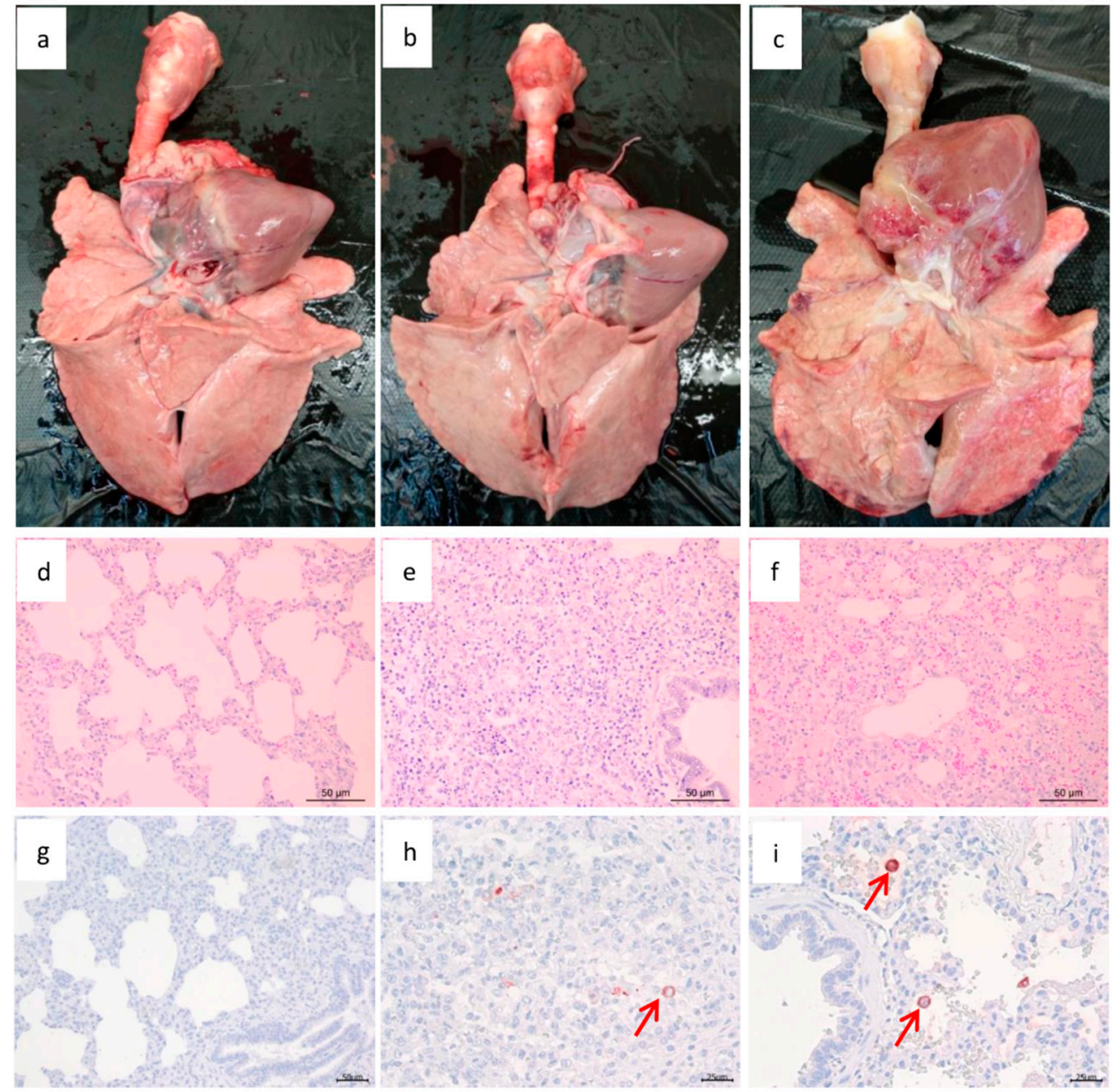

Figure 6. Lung gross lesion, histopathological and immunohistochemical examinations. Lung consolidation and extensive hemorrhages were observed in XJ17-5-infected pigs but not in JSTZ1712-12-infected or mock-infected pigs (a-c). In the histopathological examination, red blood cells and serous exudation were obviously observed only in XJ17-5-infected pigs (f). In addition, interstitial pneumonia with infiltration of lymphocytes and macrophages could also be observed in the lungs of XJ17-5-infected and JSTZ1712-12-infected pigs but not in mock-infected pigs (d-f). PRRSV antigens could be detected in both JSTZ1712-12-infected and XJ17-5-infected pigs but not in mock-infected pigs in the immunohistochemical examination $(\mathbf{g}-\mathbf{i})$. The red arrows point the positive PRRSV antigen.

\subsection{Potential Virulence-Associated Amino Acids}

To further evaluate the amino acid changes potentially related to the distinct virulence of XJ17-5 and JSTZ1712-12 isolates, the amino acid differences between XJ17-5 and JSTZ1712-12 were analyzed. Totally 34 differences were identified, including 28 differences within nonstructural proteins $(n s p 1 \alpha$, $n s p 1 \beta, n s p 2, n s p 9$ and $n s p 10)$ and 6 differences within structural proteins (GP3, GP4, GP5 and N) (Table 2). Notably, 21 out of the 34 differences were unique for high virulent XJ17-5 isolate and differed from avirulent JSTZ1712-12 isolate and the other 9 highly homologous HP-PRRSV variants available in GenBank database. A majority of these differences (19 out of 21) in the 9 other HP-PRRSV variants are exactly identical to the avirulent JSTZ1712-12 isolates. 
Table 2. Distinct amino acids between XJ17-5 and JSTZ1712-12 isolates and corresponding sites in 9 other highly homologous highly pathogenic (HP)-PRRSV variants.

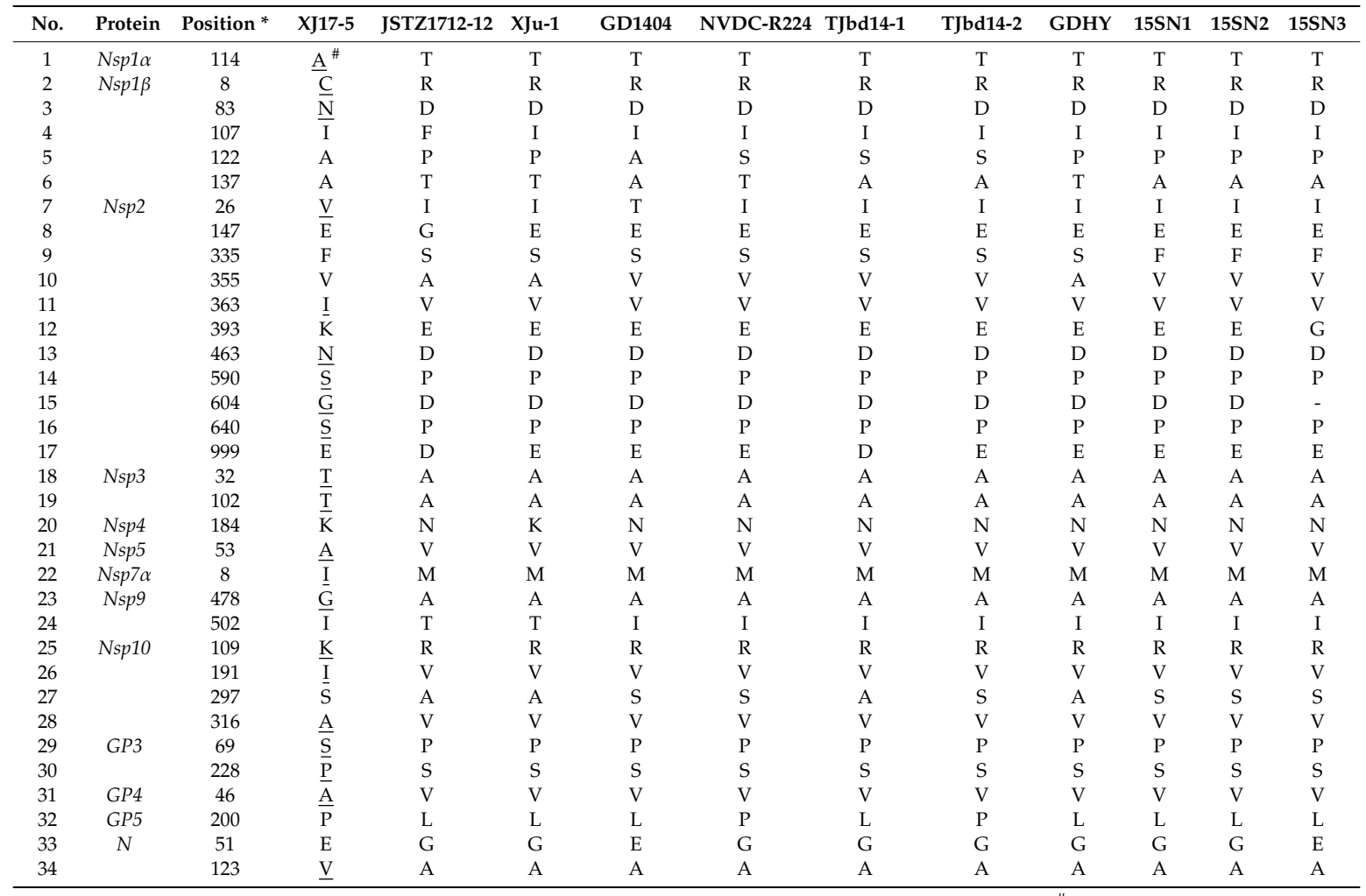

* The positions are determined based on each encoded protein of XJ17-5 isolate (MK759853). \# The animo acids that are unique in XJ17-5 and differ from all the other 10 highly homologous HP-PRRSV variants are shown in bold and underlined.

\section{Discussion}

HP-PRRS pandemics caused by HP-PRRSV have seriously influenced the development of the Chinese swine industry since 2006 [11,14]. Even though several control strategies, including the commercial modified live HP-PRRS vaccines, have been widely used, HP-PRRS outbreaks in Chinese swine herds are still not effectively controlled due to the continuous emergence of novel HP-PRRSV variants and limited cross-protection of commercial vaccines against heterologous isolates [6,32-34]. In this study, we identified two novel HP-PRRSV variants (XJ17-5 and JSTZ1712-12) that have the new genetic feature of 150-amino-acid deletion in $n s p 2$. These new HP-PRRSV variants form a new branch within the HP-PRRSV subtype. Even though XJ17-5 and JSTZ1712-12 isolates share high genomic homology, they have distinct pathogenicity for piglets. The amino acid differences between XJ17-5 and JSTZ1712-12 isolates that are potentially associated with the distinct virulence were also determined.

Nsp2 contains three major domains: a papain-like protease domain (PLP2) at N terminus, a 500to 700-amino-acid middle hypervariable region and a C-terminal transmembrane domain [35]. Even though $n s p 2$ is one of the most variable PRRSV regions, the protease domain and transmembrane domain in $n s p 2$ are much more conserved than the middle hypervariable region. The size of $n s p 2$ is quite variable due to the natural deletion and insertion in the middle hypervariable region $[11,12,36,37]$. The discontinuous 30-amino-acid deletion (positions 481 and 532-560) in $n s p 2$ is the genetic marker of HP-PRRSV isolates; however, this unique deletion in $n s p 2$ is not related to the high virulence of HP-PRRSV [16]. Notably, our isolates have same additional 120 amino acid deletion at positions 628 to 747 of $n s p 2$. BLAST and phylogenetic analyses showed that at least 11 highly homologous viruses with the new genetic feature have been isolated in different regions of China from 2012 to 2017 [38-40]. These variants form a new branch within the HP-PRRSV subtype, suggesting that a new group of 
HP-PRRSV variants is circulating in Chinese swine herds for a long time. However, the virulence of these new variants was not determined yet.

Different PRRSV isolates have distinct pathogenicity. In general, HP-PRRSV isolates have the highest virulence followed by NADC30-like and classical PRRSV2 isolates $[11,17,19,41]$, while current Chinese PRRSV1 isolates are low virulent [8,42]. Retrospective study showed that our XJ17-5 and JSTZ1712-12 isolates were obtained from clinically diseased and healthy pigs, respectively. Whether these highly homologous HP-PRRSV variants with novel deletion in $n s p 2$ have distinct virulence becomes an interesting question. Notably, a recent study identified that a new isolate JX2014T2, which contains the same 150-amino-acid deletion in $n s p 2$, is highly pathogenic to piglets [24]. However, the similarity between the JX2014T2 and our isolates was unable to be determined because the JX2014T2 sequence is not available. Our challenge study showed that XJ17-5 caused high fever, $100 \%$ morbidity and $60 \%$ mortality, which is similar to the typical HP-PRRSV isolates [11,43]. However, JSTZ1712-12 did not cause fever, any obvious clinical sign or death during the challenge study, suggesting that JSTZ1712-12 is avirulent in piglet. These results showed that even though XJ17-5 and JSTZ1712-12 share high genomic identity, they have significantly distinct virulence. A previous study showed that a mutant with 131-amino-acid deletion (positions 628-759) in nsp2 of P129 strain is less virulent than the parental virus in pigs [36]. The deletion in the mutant is largely overlapped with the natural 120 -amino-acid deletion in our isolates, which raises a hypothesis that the 120-amino-acid deletion in $n s p 2$ may be related to the virulence. However, the existence of this deletion in both high virulence and avirulence isolates suggests that the deletion is probably not associated with the virulence.

To analyze the virulence determinants of PRRSV isolates, two methods including sequence comparison and reverse genetic manipulation are commonly used [44]. Several studies compared the genomic sequences of high virulence parental virus and the attenuated vaccine strain obtained from in vitro passage in Marc-145 cells to identify the potential mutations associated with the attenuation [15,45-47]. The obtained mutations might be related to virulence determinants; however, they might also be associated with the viral adaptation to Marc-145 cells [48,49]. We identified that there are 34 amino acid differences between XJ17-5 and JSTZ1712-12 isolates. These amino acid changes are not related to in vitro adaptation; therefore, they are more likely correlated with virulence determinants. Due to the limited amounts of samples and the low virus loads in the samples, the third passages of XJ17-5 and JSTZ1712-12 isolates rather than the original samples were used for complete genome sequencing and animal challenge study. Previous studies have proved that few passages will not cause significant changes in either the virulence or the genome of PRRSV [15,47].

Plenty of studies have been performed using the reverse genetics to exchange different genes/regions between two PRRSV isolates that are genetically and pathologically distinct $[16,50,51]$. A concern about this approach is that virulence determinants of PRRSV isolates are likely strain-specific [44,52-54], the virulence determinants for one parental virus might be not the same for the other genetically distinct parental virus. The identification of natural HP-PRRSV variants with high genomic similarity but distinct virulence provides ideal viruses to analyze the virulence determinants of these HP-PRRSV variants. A previous study reported that $n s p 9$ and $n s p 10$ contribute to the fatal virulence of HP-PRRSV [51]. Furthermore, two recent studies identified mutations in $n s p 9$ that play crucial roles in the replication and pathogenicity of HP-PRRSV $[55,56]$. In this study, two amino acid differences in $n s p 9\left(\mathrm{G}_{478} \mathrm{~A}\right.$ and $\left.\mathrm{I}_{502} \mathrm{~T}\right)$ and four amino acid differences in $n s p 10\left(\mathrm{~K}_{109} \mathrm{R}, \mathrm{I}_{191} \mathrm{~V}, \mathrm{~S}_{297} \mathrm{~A}\right.$ and $\mathrm{A}_{316} \mathrm{~V}$ ) were identified between XJ17-5 and JSTZ1712-12 isolates (Table 2). These amino acid sites are not consistent with the virulence-associated mutations identified in previous studies $[51,55,56]$. Considering that XJ17-5 and JSTZ1712-12 isolates do not have significant difference in in vitro and in vivo replication (Figures 2 and 5c), the amino acid differences in $n s p 9$ and $n s p 10$ identified in this study might be not associated with the replication efficiency or the pathogenicity, suggesting that the differences in other regions may also play roles of virulence determinants. For example, Nsp3-8 and ORF5 were reported to contain major virulence determinants [50], the mutations in these regions identified in our study might be associated with distinct virulence. Notably, a majority of the mutations 
(21 out of 34) spread throughout the genome are unique for high virulent XJ17-5 isolate, and 19 out of the 21 mutations are identical in avirulent JSTZ1712-12 and 9 other high homologous HP-PRRSV variants identified by other research groups. Therefore, it is rational to speculate that these mutations have higher probability to associate with the virulence. The infectious clones of XJ17-5 and JSTZ1712-12 isolates have been successfully constructed and rescued in our laboratory. Chimeric and mutant viruses would be constructed to determine the virulence-associated amino acids in the near future.

\section{Conclusions}

In conclusion, this study isolated two HP-PRRSV variants from a severe abortion farm and a clinically healthy farm in China 2017. The two isolates (XJ17-5 and JSTZ1712-12) share 99.45\% genomic identity and both contain a 30-amino-acid discontinuous deletion and a 120-amino-acid continuous deletion in $n s p 2$. Genome-based phylogenetic analysis showed that they belong to HP-PRRSV subtype but form a new branch with other HP-PRRSV variants containing the same 150-amino-acid deletion in $n s p 2$. Pathogenic analysis showed that XJ17-5 is high virulence causing high fever and $60 \%$ mortality, while JSTZ1712-12 is avirulent. Fragment comparisons identified 34 amino acid differences between XJ17-5 and JSTZ1712-12 isolates that might be related to distinct virulence. This study identified highly homologous HP-PRRSV variants with distinct virulence, which contributes to further analyze the pathogenesis and evolution of PRRSV in the field.

Supplementary Materials: The following are available online at http://www.mdpi.com/1999-4915/11/9/875/s1, Table S1: Primers used for PRRSV2 genome amplification. Table S2. AUC values for virus load versus time in PAM and Marc-145 cells.

Author Contributions: Conceptualization, N.C.; data curation, N.C., X.L. and X.Y.; formal analysis, N.C., X.L., K.T. and J.Z.; funding acquisition, N.C. and J.Z.; investigation, N.C., M.Y., Y.H., S.L., Y.X., X.L. and S.L.; methodology, N.C., M.Y., Y.H., S.L. and Y.X.; resources, N.C., K.T. and J.Z.; supervision, J.Z.; validation, N.C. and J.Z.; writing—original draft, N.C.; writing-review and editing, X.L. and J.Z.

Funding: This research was funded by National Natural Science Foundation of China (31802172), Natural Science Foundation of Jiangsu Province (BK20170492), China Postdoctoral Science Foundation (2016M590510), Jiangsu Co-innovation Center for Prevention and Control of Important Animal Infectious Diseases and Zoonoses, and the Priority Academic Program Development of Jiangsu Higher Education Institutions (PAPD).

Acknowledgments: The authors would like to thank the staff at Experimental Animal Center of Yangzhou University for their help in handling our animals. Nanhua Chen is supported by High Talent Supporting Program of Yangzhou University.

Conflicts of Interest: The authors declare no conflict of interest. The funders had no role in the design of the study; in the collection, analyses, or interpretation of data; in the writing of the manuscript, or in the decision to publish the results.

\section{References}

1. Holtkamp, D.J.; Kliebenstein, J.B.; Neumann, E.J.; Zimmerman, J.J.; Rotto, H.F.; Yoder, T.K.; Wang, C.; Yeske, P.E.; Mowrer, C.L.; Haley, C.A. Assessment of the economic impact of porcine reproductive and respiratory syndrome virus on United States pork producers. J. Swine Health Prod. 2013, 21, 72-84.

2. Keffaber, K.K. Reprodutive failure of unknown etiology. Am. Assoc. Swine Vet. 1989, 1, 1-10.

3. Lunney, J.K.; Fang, Y.; Ladinig, A.; Chen, N.; Li, Y.; Rowland, B.; Renukaradhya, G.J. Porcine Reproductive and Respiratory Syndrome Virus (PRRSV): Pathogenesis and Interaction with the Immune System. Annu. Rev. Anim. Biosci. 2016, 4, 129-154. [CrossRef] [PubMed]

4. Fang, Y.; Treffers, E.E.; Li, Y.; Tas, A.; Sun, Z.; van der Meer, Y.; de Ru, A.H.; van Veelen, P.A.; Atkins, J.F.; Snijder, E.J.; et al. Efficient -2 frameshifting by mammalian ribosomes to synthesize an additional arterivirus protein. Proc. Natl. Acad. Sci. USA 2012, 109, E2920-E2928. [CrossRef] [PubMed]

5. Normile, D. Virology. China, Vietnam grapple with 'rapidly evolving' pig virus. Science 2007, $317,1017$. [CrossRef] [PubMed]

6. Yu, X.; Chen, N.; Wang, L.; Wu, J.; Zhou, Z.; Ni, J.; Li, X.; Zhai, X.; Shi, J.; Tian, K. New genomic characteristics of highly pathogenic porcine reproductive and respiratory syndrome viruses do not lead to significant changes in pathogenicity. Vet. Microbiol. 2012, 158, 291-299. [CrossRef] [PubMed] 
7. Adams, M.J.; Lefkowitz, E.J.; King, A.M.Q.; Harrach, B.; Harrison, R.L.; Knowles, N.J.; Kropinski, A.M.; Krupovic, M.; Kuhn, J.H.; Mushegian, A.R.; et al. Changes to taxonomy and the International Code of Virus Classification and Nomenclature ratified by the International Committee on Taxonomy of Viruses (2017). Arch. Virol. 2017, 162, 2505-2538. [CrossRef]

8. Chen, N.; Cao, Z.; Yu, X.; Deng, X.; Zhao, T.; Wang, L.; Liu, Q.; Li, X.; Tian, K. Emergence of novel European genotype porcine reproductive and respiratory syndrome virus in mainland China. J. Gen. Virol. 2011, 92, 880-892. [CrossRef]

9. Chen, N.; Huang, Y.; Ye, M.; Li, S.; Xiao, Y.; Cui, B.; Zhu, J. Co-infection status of classical swine fever virus (CSFV), porcine reproductive and respiratory syndrome virus (PRRSV) and porcine circoviruses (PCV2 and PCV3) in eight regions of China from 2016 to 2018. Infect. Genet. Evol. 2019, 68, 127-135. [CrossRef]

10. Guo, B.; Chen, Z.; Liu, W.; Cui, Y. Isolation and identification of porcine reproductory and respiratory syndrome (PRRS) virus from aborted fetuses suspected of PRRS. Chin. J. Prev. Vet. Med. 1996, 2, 1-5.

11. Tian, K.; Yu, X.; Zhao, T.; Feng, Y.; Cao, Z.; Wang, C.; Hu, Y.; Chen, X.; Hu, D.; Tian, X.; et al. Emergence of fatal PRRSV variants: Unparalleled outbreaks of atypical PRRS in China and molecular dissection of the unique hallmark. PLoS ONE 2007, 2, e526. [CrossRef] [PubMed]

12. Zhao, K.; Ye, C.; Chang, X.B.; Jiang, C.G.; Wang, S.J.; Cai, X.H.; Tong, G.Z.; Tian, Z.J.; Shi, M.; An, T.Q. Importation and Recombination Are Responsible for the Latest Emergence of Highly Pathogenic Porcine Reproductive and Respiratory Syndrome Virus in China. J. Virol. 2015, 89, 10712-10716. [CrossRef] [PubMed]

13. Li, H.; Yang, H. Infection of porcine reproductive and respiratory syndrome virus suppresses the antibody response to classical swine fever virus vaccination. Vet. Microbiol. 2003, 95, 295-301. [CrossRef]

14. Tong, G.Z.; Zhou, Y.J.; Hao, X.F.; Tian, Z.J.; An, T.Q.; Qiu, H.J. Highly pathogenic porcine reproductive and respiratory syndrome, China. Emerg. Infect. Dis. 2007, 13, 1434-1436. [CrossRef] [PubMed]

15. Leng, X.; Li, Z.; Xia, M.; Li, X.; Wang, F.; Wang, W.; Zhang, X.; Wu, H. Mutations in the genome of the highly pathogenic porcine reproductive and respiratory syndrome virus potentially related to attenuation. Vet. Microbiol. 2012, 157, 50-60. [CrossRef] [PubMed]

16. Zhou, L.; Zhang, J.; Zeng, J.; Yin, S.; Li, Y.; Zheng, L.; Guo, X.; Ge, X.; Yang, H. The 30-amino-acid deletion in the Nsp2 of highly pathogenic porcine reproductive and respiratory syndrome virus emerging in China is not related to its virulence. J. Virol. 2009, 83, 5156-5167. [CrossRef] [PubMed]

17. Sun, Z.; Wang, J.; Bai, X.; Ji, G.; Yan, H.; Li, Y.; Wang, Y.; Tan, F.; Xiao, Y.; Li, X.; et al. Pathogenicity comparison between highly pathogenic and NADC30-like porcine reproductive and respiratory syndrome virus. Arch. Virol. 2016, 161, 2257-2261. [CrossRef] [PubMed]

18. Zhou, L.; Wang, Z.; Ding, Y.; Ge, X.; Guo, X.; Yang, H. NADC30-like Strain of Porcine Reproductive and Respiratory Syndrome Virus, China. Emerg. Infect. Dis. 2015, 21, 2256-2257. [CrossRef] [PubMed]

19. Brockmeier, S.L.; Loving, C.L.; Vorwald, A.C.; Kehrli, M.E., Jr.; Baker, R.B.; Nicholson, T.L.; Lager, K.M.; Miller, L.C.; Faaberg, K.S. Genomic sequence and virulence comparison of four Type 2 porcine reproductive and respiratory syndrome virus strains. Virus Res. 2012, 169, 212-221. [CrossRef]

20. Chen, N.; Li, S.; Zhou, R.; Zhu, M.; He, S.; Ye, M.; Huang, Y.; Li, S.; Zhu, C.; Xia, P.; et al. Two novel porcine epidemic diarrhea virus (PEDV) recombinants from a natural recombinant and distinct subtypes of PEDV variants. Virus Res. 2017, 242, 90-95. [CrossRef]

21. Chen, N.; Ye, M.; Xiao, Y.; Li, S.; Huang, Y.; Li, X.; Tian, K.; Zhu, J. Development of universal and quadruplex real-time RT-PCR assays for simultaneous detection and differentiation of porcine reproductive and respiratory syndrome viruses. Transbound. Emerg. Dis. 2019. [CrossRef] [PubMed]

22. Chen, N.H.; Chen, X.Z.; Hu, D.M.; Yu, X.L.; Wang, L.L.; Han, W.; Wu, J.J.; Cao, Z.; Wang, C.B.; Zhang, Q.; et al. Rapid differential detection of classical and highly pathogenic North American Porcine Reproductive and Respiratory Syndrome virus in China by a duplex real-time RT-PCR. J. Virol. Methods 2009, 161, 192-198. [CrossRef] [PubMed]

23. Chen, N.; Ye, M.; Li, S.; Huang, Y.; Zhou, R.; Yu, X.; Tian, K.; Zhu, J. Emergence of a novel highly pathogenic recombinant virus from three lineages of porcine reproductive and respiratory syndrome virus 2 in China 2017. Transbound. Emerg. Dis. 2018, 65, 1775-1785. [CrossRef] [PubMed]

24. Yu, L.X.; Wang, X.; Yu, H.; Jiang, Y.F.; Gao, F.; Tong, W.; Li, L.W.; Li, H.C.; Yang, S.; Chen, P.F.; et al. The emergence of a highly pathogenic porcine reproductive and respiratory syndrome virus with additional 120aa deletion in Nsp2 region in Jiangxi, China. Transbound. Emerg. Dis. 2018, 65, 1740-1748. [CrossRef] [PubMed] 
25. Larkin, M.A.; Blackshields, G.; Brown, N.P.; Chenna, R.; McGettigan, P.A.; McWilliam, H.; Valentin, F.; Wallace, I.M.; Wilm, A.; Lopez, R.; et al. Clustal W and Clustal X version 2.0. Bioinformatics 2007, 23, 2947-2948. [CrossRef] [PubMed]

26. Tamura, K.; Stecher, G.; Peterson, D.; Filipski, A.; Kumar, S. MEGA6: Molecular Evolutionary Genetics Analysis version 6.0. Mol. Biol. Evol. 2013, 30, 2725-2729. [CrossRef] [PubMed]

27. Chen, N.; Liu, Q.; Qiao, M.; Deng, X.; Chen, X.; Sun, M. Whole genome characterization of a novel porcine reproductive and respiratory syndrome virus 1 isolate: Genetic evidence for recombination between Amervac vaccine and circulating strains in mainland China. Infect. Genet. Evol. 2017, 54, 308-313. [CrossRef] [PubMed]

28. Lole, K.S.; Bollinger, R.C.; Paranjape, R.S.; Gadkari, D.; Kulkarni, S.S.; Novak, N.G.; Ingersoll, R.; Sheppard, H.W.; Ray, S.C. Full-length human immunodeficiency virus type 1 genomes from subtype C-infected seroconverters in India, with evidence of intersubtype recombination. J. Virol. 1999, 73, 152-160.

29. Martin, D.P.; Murrell, B.; Golden, M.; Khoosal, A.; Muhire, B. RDP4: Detection and analysis of recombination patterns in virus genomes. Virus Evol. 2015, 1, vev003. [CrossRef]

30. Kroll, J.; Piontkowski, M.; Kraft, C.; Coll, T.; Gomez-Duran, O. Initial vaccination and revaccination with Type I PRRS $94881 \mathrm{MLV}$ reduces viral load and infection with porcine reproductive and respiratory syndrome virus. Porc. Health Manag. 2018, 4, 23. [CrossRef]

31. Ooms, K.; van Gorp, H.; Botti, S.; van Gaever, T.; Delputte, P.L.; Nauwynck, H.J. Evaluation of viral peptide targeting to porcine sialoadhesin using a porcine reproductive and respiratory syndrome virus vaccination-challenge model. Virus Res. 2013, 177, 147-155. [CrossRef] [PubMed]

32. Shi, M.; Holmes, E.C.; Brar, M.S.; Leung, F.C. Recombination is associated with an outbreak of novel highly pathogenic porcine reproductive and respiratory syndrome viruses in China. J. Virol. 2013, 87, 10904-10907. [CrossRef]

33. Bai, X.; Wang, Y.; Xu, X.; Sun, Z.; Xiao, Y.; Ji, G.; Li, Y.; Tan, F.; Li, X.; Tian, K. Commercial vaccines provide limited protection to NADC30-like PRRSV infection. Vaccine 2016, 34, 5540-5545. [CrossRef] [PubMed]

34. Zhou, L.; Yang, B.; Xu, L.; Jin, H.; Ge, X.; Guo, X.; Han, J.; Yang, H. Efficacy evaluation of three modified-live virus vaccines against a strain of porcine reproductive and respiratory syndrome virus NADC30-like. Vet. Microbiol. 2017, 207, 108-116. [CrossRef] [PubMed]

35. Sun, Z.; Li, Y.; Ransburgh, R.; Snijder, E.J.; Fang, Y. Nonstructural protein 2 of porcine reproductive and respiratory syndrome virus inhibits the antiviral function of interferon-stimulated gene 15. J. Virol. 2012, 86, 3839-3850. [CrossRef]

36. Kim, D.Y.; Kaiser, T.J.; Horlen, K.; Keith, M.L.; Taylor, L.P.; Jolie, R.; Calvert, J.G.; Rowland, R.R. Insertion and deletion in a non-essential region of the nonstructural protein $2(n s p 2)$ of porcine reproductive and respiratory syndrome (PRRS) virus: Effects on virulence and immunogenicity. Virus Genes 2009, 38, 118-128. [CrossRef]

37. Yoshii, M.; Okinaga, T.; Miyazaki, A.; Kato, K.; Ikeda, H.; Tsunemitsu, H. Genetic polymorphism of the nsp2 gene in North American type-porcine reproductive and respiratory syndrome virus. Arch. Virol. 2008, 153, 1323-1334. [CrossRef] [PubMed]

38. Xia, J.; Chen, S.; Huang, J.; Ma, W.; Du, W.; Yang, X.; Liu, Y.; Yan, G. Complete genomic characterization of a porcine reproductive and respiratory syndrome virus isolate in Xinjiang province of China. Virus Genes 2015, 50, 39-45. [CrossRef]

39. Yu, L.; Zhao, P.; Dong, J.; Liu, Y.; Zhang, L.; Liang, P.; Wang, L.; Song, C. Genetic characterization of 11 porcine reproductive and respiratory syndrome virus isolates in South China from 2014 to 2015. Virol. J. 2017, 14, 139. [CrossRef]

40. Zhao, H.; Han, Q.; Zhang, L.; Zhang, Z.; Wu, Y.; Shen, H.; Jiang, P. Emergence of mosaic recombinant strains potentially associated with vaccine JXA1-R and predominant circulating strains of porcine reproductive and respiratory syndrome virus in different provinces of China. Virol. J. 2017, 14, 67. [CrossRef]

41. Li, B.; Fang, L.; Xu, Z.; Liu, S.; Gao, J.; Jiang, Y.; Chen, H.; Xiao, S. Recombination in vaccine and circulating strains of porcine reproductive and respiratory syndrome viruses. Emerg. Infect. Dis. 2009, 15, 2032-2035. [CrossRef] [PubMed]

42. Ming, S.; Yongying, M.; Bohua, L.; Huiying, L.; Xiaoyu, D.; Qiaorong, L.; Mingming, Q.; Xi, C.; Xinyan, Y.; Xizhao, C. Pathogenic Characterization of European Genotype Porcine Reproductive and Respiratory Syndrome Virus Recently Isolated in Mainland China. Open Virol. J. 2017, 11, 83-89. [CrossRef] [PubMed] 
43. Zhang, H.; Xia, M.; Wang, W.; Ju, D.; Cao, L.; Wu, B.; Wang, X.; Wu, Y.; Song, N.; Hu, J.; et al. An Attenuated Highly Pathogenic Chinese PRRS Viral Vaccine Confers Cross Protection to Pigs against Challenge with the Emerging PRRSV NADC30-Like Strain. Virol. Sin. 2018, 33, 153-161. [CrossRef]

44. Han, J.; Zhou, L.; Ge, X.; Guo, X.; Yang, H. Pathogenesis and control of the Chinese highly pathogenic porcine reproductive and respiratory syndrome virus. Vet. Microbiol. 2017, 209, 30-47. [CrossRef] [PubMed]

45. Allende, R.; Kutish, G.F.; Laegreid, W.; Lu, Z.; Lewis, T.L.; Rock, D.L.; Friesen, J.; Galeota, J.A.; Doster, A.R.; Osorio, F.A. Mutations in the genome of porcine reproductive and respiratory syndrome virus responsible for the attenuation phenotype. Arch. Virol. 2000, 145, 1149-1161. [CrossRef]

46. Kim, W.I.; Kim, J.J.; Cha, S.H.; Yoon, K.J. Different biological characteristics of wild-type porcine reproductive and respiratory syndrome viruses and vaccine viruses and identification of the corresponding genetic determinants. J. Clin. Microbiol. 2008, 46, 1758-1768. [CrossRef]

47. Han, W.; Wu, J.J.; Deng, X.Y.; Cao, Z.; Yu, X.L.; Wang, C.B.; Zhao, T.Z.; Chen, N.H.; Hu, H.H.; Bin, W.; et al. Molecular mutations associated with the in vitro passage of virulent porcine reproductive and respiratory syndrome virus. Virus Genes 2009, 38, 276-284. [CrossRef] [PubMed]

48. Zhang, H.L.; Tang, Y.D.; Liu, C.X.; Xiang, L.R.; Zhang, W.L.; Leng, C.L.; Wang, Q.; An, T.Q.; Peng, J.M.; Tian, Z.J.; et al. Adaptions of field PRRSVs in Marc-145 cells were determined by variations in the minor envelope proteins GP2a-GP3. Vet. Microbiol. 2018, 222, 46-54. [CrossRef] [PubMed]

49. Xie, J.; Trus, I.; Oh, D.; Kvisgaard, L.K.; Rappe, J.C.F.; Ruggli, N.; Vanderheijden, N.; Larsen, L.E.; Lefevre, F.; Nauwynck, H.J. A Triple Amino Acid Substitution at Position 88/94/95 in Glycoprotein GP2a of Type 1 Porcine Reproductive and Respiratory Syndrome Virus (PRRSV1) Is Responsible for Adaptation to MARC-145 Cells. Viruses 2019, 11, 36. [CrossRef] [PubMed]

50. Kwon, B.; Ansari, I.H.; Pattnaik, A.K.; Osorio, F.A. Identification of virulence determinants of porcine reproductive and respiratory syndrome virus through construction of chimeric clones. Virology 2008, 380, 371-378. [CrossRef]

51. Li, Y.; Zhou, L.; Zhang, J.; Ge, X.; Zhou, R.; Zheng, H.; Geng, G.; Guo, X.; Yang, H. Nsp9 and nsp10 contribute to the fatal virulence of highly pathogenic porcine reproductive and respiratory syndrome virus emerging in china. PLoS Pathog. 2014, 10, e1004216. [CrossRef] [PubMed]

52. Osorio, F.A.; Galeota, J.A.; Nelson, E.; Brodersen, B.; Doster, A.; Wills, R.; Zuckermann, F.; Laegreid, W.W. Passive transfer of virus-specific antibodies confers protection against reproductive failure induced by a virulent strain of porcine reproductive and respiratory syndrome virus and establishes sterilizing immunity. Virology 2002, 302, 9-20. [CrossRef] [PubMed]

53. Lager, K.M.; Mengeling, W.L.; Wesley, R.D. Strain predominance following exposure of vaccinated and naive pregnant gilts to multiple strains of porcine reproductive and respiratory syndrome virus. Can. J. Vet. Res. 2003, 67, 121-127. [PubMed]

54. Mengeling, W.L.; Lager, K.M.; Vorwald, A.C.; Koehler, K.J. Strain specificity of the immune response of pigs following vaccination with various strains of porcine reproductive and respiratory syndrome virus. Vet. Microbiol. 2003, 93, 13-24. [CrossRef]

55. Xu, L.; Zhou, L.; Sun, W.; Zhang, P.; Ge, X.; Guo, X.; Han, J.; Yang, H. Nonstructural protein 9 residues 586 and 592 are critical sites in determining the replication efficiency and fatal virulence of the Chinese highly pathogenic porcine reproductive and respiratory syndrome virus. Virology 2018, 517, 135-147. [CrossRef] [PubMed]

56. Zhao, K.; Gao, J.C.; Xiong, J.Y.; Guo, J.C.; Yang, Y.B.; Jiang, C.G.; Tang, Y.D.; Tian, Z.J.; Cai, X.H.; Tong, G.Z.; et al. Two Residues in NSP9 Contribute to the Enhanced Replication and Pathogenicity of Highly Pathogenic Porcine Reproductive and Respiratory Syndrome Virus. J. Virol. 2018, 92. [CrossRef] [PubMed]

(C) 2019 by the authors. Licensee MDPI, Basel, Switzerland. This article is an open access article distributed under the terms and conditions of the Creative Commons Attribution (CC BY) license (http://creativecommons.org/licenses/by/4.0/). 